\title{
Development of Molecular Recognition Membrane showing Autonomous Adsorption-Desorption Phenomenon
}

\author{
Hidenori Ohashi $^{\dagger}$, Toshio Shimada ${ }^{\ddagger}$, and Takeo Yamaguchi ${ }^{\dagger}$ \\ ${ }^{\dagger}$ Chemical Resources Laboratory, Tokyo Institute of Technology, \\ R1-17 4259, Nagatsuta-cho, Midori-ku, Yokohama-city, Kanagawa 226-8503, Japan \\ ${ }^{\ddagger}$ Department of Chemical System Engineering, The University of Tokyo, \\ 7-3-1, Hongo, Bunkyo-ku, Tokyo 113-8656, Japan
}

Key words: molecular recognition, autonomous oscillation, plasma graft polymerization

\section{Introduction}

In biomembranes, distinct processes such as structural change and mass transport, are highly regulated by the coordination phenomenon induced by molecular recognition. This enables the living body to create rhythmical nonlinear oscillatory phenomena, such as circadian rhythm, autonomic heart beat, and brain wave. By seeking such chemical oscillation system, some researchers achieved artificial nonlinear oscillators such as BZ reaction, permeation phenomena of membrane, rhythmic drug delivery systems, and so on.[1-5] However, only one work has achieved an artificial oscillatory system induced by molecular recognition, the important feature of living body.[6]

Meanwhile, our group has succeeded in developing polymeric material exhibiting a coordination of molecular recognition and actuation function within the polymer itself.[7] This was the first synthetic material showing nonlinear coordination behavior induced by molecular recognition. This polymer material is a linear copolymer of $N$-isopropylacrylamide (NIPAM) and $\beta$-cyclo dextrin ( $\beta$-CD) with high CD content as shown in Figure 1. NIPAM is a well-known thermosensitive polymer that exhibits reversible swelling/shrinking phase transition in response to temperature change. The $\beta$-CD moiety is a bucket-like oligosaccharide and acts as a molecular host that can capture a specific molecule of 8-anilino-1-naphthalene sulfuric acid ammonium salt (ANS) as a guest molecule.

In the copolymer, NIPAM moiety and CD/ANS complex affect each other. Swelling/shrinking of NIPAM polymer affects CD/ANS complex forma-

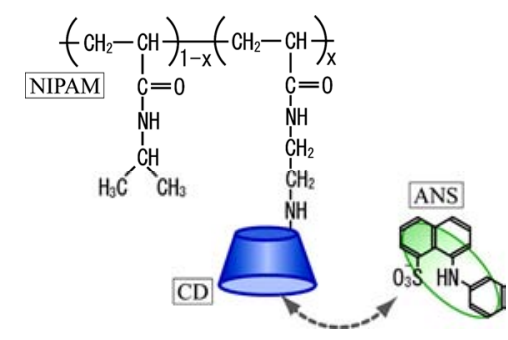

Figure 1. Schematic structures of poly(NIPAM-co- $\beta$ $\mathrm{CD}$ ) and ANS as a guest of CD.

tion through steric hinderance around the complex. On the other hand, NIPAM swelling/shrinking behavior is induced by hydrophobicity change of CD/ANS through complexation/decomplexation. These mutual effects can bring coordination behavior of NIPAM swelling/shrinking associating with CD/ANS complexation/decomplexation. As shown in Figure 2(a), triggering drastic temperature increase induces (1) "polymer shrinking” first, then steric hindrance by shrunk polymer prompts (2) “CD/ANS decomplexation." After that hydrophilicity increase by the decomplexation induces (3) "polymer swelling," and resulting relaxation of steric hinderance prompts (4) "CD/ANS recomplexation." Detailed mechanism of the distinct phenomenon can be found in the previous work.[7]

However, in the previous study, the phenomenon proceeded only one cycle, because the system was closed system of polymer solution. After one cycle, the system drops into equilibrium. In order to circumvent this insufficiency and to proceed more than one cycle as shown in Figure 2(b), develop-

\footnotetext{
Received April 2, 2009

Accepted May 8, 2009
} 


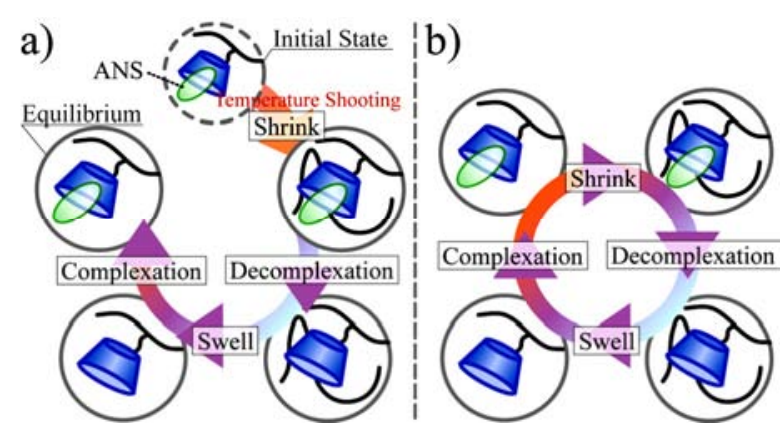

Figure 2. Schematic of coordinative oscillatory phenomenon in (a) closed polymer solution system, and (b) open membrane system of poly(NIPAM$c o-\beta-C D) / A N S$ system.

ment of open system is required. Poly(NIPAM$c o-\beta-\mathrm{CD})$ grafted porous membrane is a good candidate of such open system, because concentration gradient through membrane can easily realize successive energy inlet.

Introduction of sufficient CD moiety and elimination of comonomer other than NIPAM and CD are crucial also in the membrane system, because nonlinear coordination requires sufficient mutual effects between NIPAM and CD/ANS. A previous study have tried direct graft copolymerization of NIPAM and CD monomer onto porous membrane.[8] In this case, although no comonomer is introduced, introduced CD was very little due to the low reactivity of $\mathrm{CD}$ monomer. On the other hand, other previous studies have tried CD moiety substitution after preparing poly(NIPAM-co- glycidyl methacrylate) grafted membrane.[9-11] In this case, although higher content of CD moiety was attained, large amount of hydrophilic comonomer remained, which affects much NIPAM swelling/shrinking behavior. In this study, in order to obtain high CD contained with relatively low content of comonomer by designing the synthetic pathway. After that, nonlinear oscillatory phenomenon was demonstrated using the newly developed membrane.

\section{Methodology}

\subsection{Materials}

Porous polyethylene (PE) film was used as the porous substrate. The PE substrate with a thickness of $90 \mu \mathrm{m}$, a pore diameter of $200 \mathrm{~nm}$, and porosity of 50\% was kindly supplied by Asahi Chemical Co. Ltd., Japan. NIPAM was kindly provided by Kohjin Co., Tokyo, Japan, and was purified by recrystallization from $n$-hexane. Aminated $\beta$-CD was synthesized from $\beta$-CD according to reported procedures.[7] All of other materials were purchased from Wako Pure Chemicals, Osaka, Japan, and were used without any further purification.

\subsection{Introduction of Poly(NIPAM-co- $\beta$-CD) onto} membrane pore surface

Scheme 1 shows the newly proposed preparation procedure of poly(NIPAM-co- $\beta$-CD) grafted membrane. An aqueous solution of NIPAM and AAc emulsified with SDS was used as monomer solution. Both the SDS and the total monomer concentration in solution were fixed at 3.0 and $2.0 \mathrm{wt} \%$, respectively. The mole fraction of AAc in the monomers was $30 \mathrm{~mol} \%$. Plasma graft polymerization[12] was carried out to prepare the membrane having grafted poly(NIPAM-co-AAc) via peroxide technique[13] with porous PE substrate and the extensively degassed (freeze-thawed) monomer solution. After washing the prepared membrane with ethanol, carboxyl group of AAc was activated

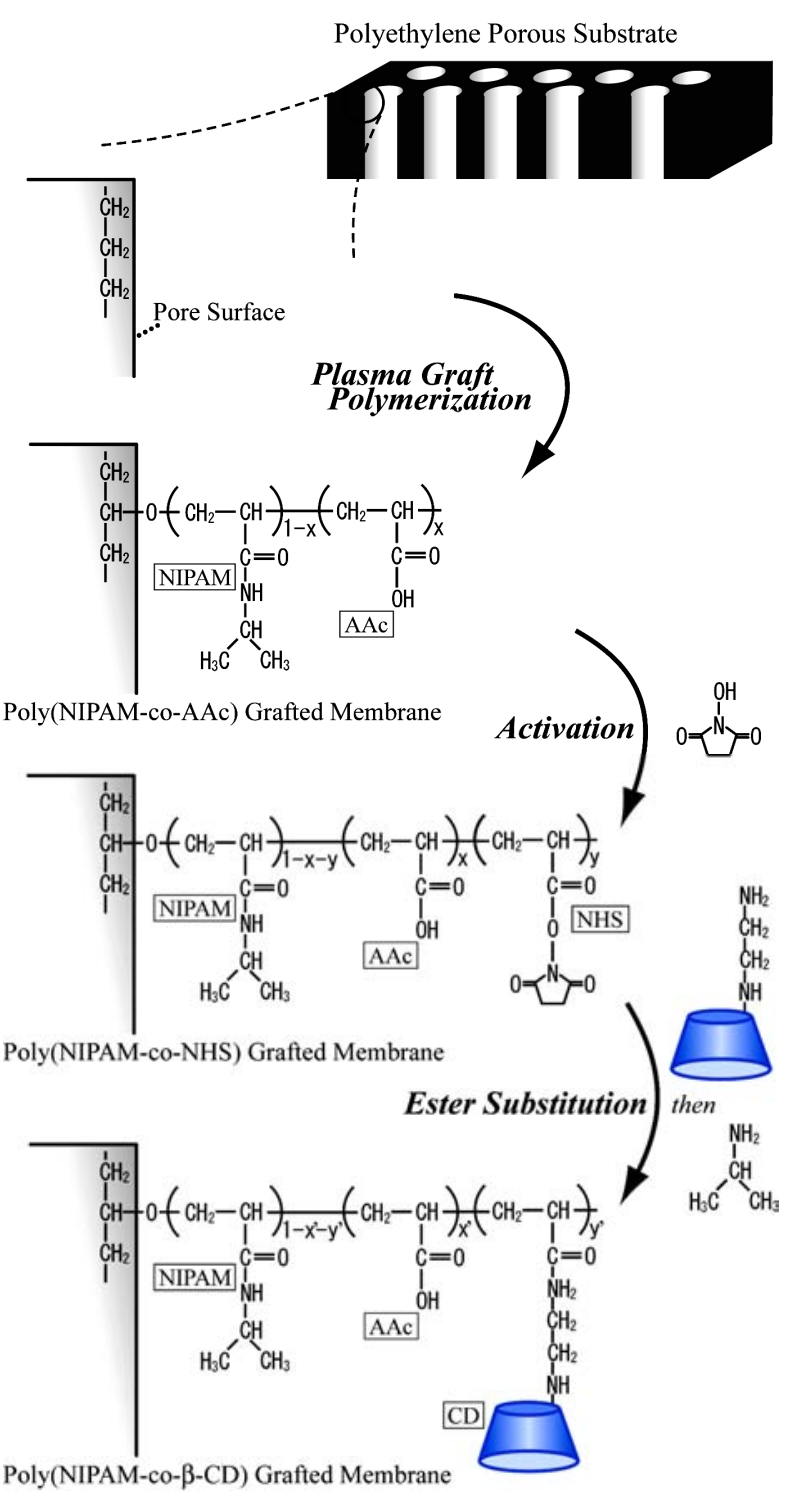

Scheme 1. A novel preparation scheme of poly(NIPAMco- $\beta$-CD) grafted porous membrane. 
using NHS (2.0 M) and WSC (2.0 M) in DMF at $25^{\circ} \mathrm{C}$ for $1 \mathrm{hr}$. After rinsing with DMF, aminated $\beta$-CD was substituted into these active esters in DMSO. Aminated $\beta$-CD was $2.0 \mathrm{wt} \%$ of total solution, and reacted at $30{ }^{\circ} \mathrm{C}$ for $120 \mathrm{hr}$. Residual active esters were substituted into NIPAM by reacting with $20 \%$ IPAm in DMF at $25^{\circ} \mathrm{C}$ for $12 \mathrm{hr}$.

The amount of NHS introduced in poly(NIPAMco-NHS) grafted membrane was determined by UV absorbance of NHS detached from the membrane after hydrolytic reaction using ammonia water. UV-vis spectrometer Model U-3310 (Hitachi, Ibaraki, Japan) and UV light of $260 \mathrm{~nm}$, which is near the maximum absorbance of NHS, is used for the test.

In order to investigate the prepared membranes, their FT-IR spectra were observed with Nicolet MAGNA550. From the height of characteristic peaks of each functional group, the introduction ratios of the monomeric units in the membrane were determined using the predetermined relationship between NMR peak area and the IR peak height ratio for linear polymer. For example, ratio of $\beta$-CD to NIPAM in the grafted polymer was calculated with following equation;

$$
\mathrm{R}=3.95 \times \frac{\text { primary alcohol of } \mathrm{CD}\left[1040 \mathrm{~cm}^{-1}\right]}{\text { acrylamideof NIPAM }\left[1550 \mathrm{~cm}^{-1}\right]}
$$

The grafting degree of the membrane, $\mathrm{W}$, is defined as $\mathrm{W}\left[\mathrm{mg} \mathrm{cm}{ }^{-2}\right]=\left(\mathrm{W}_{2}-\mathrm{W}_{1}\right) / \mathrm{A}$, where $\mathrm{W}_{1}$ is the weight of porous PE substrate before polymerization, $W_{2}$ is the weight of membrane after plasma graft polymerization, and $\mathrm{A}$ is the area of the PE substrate.

\subsection{Observation of Autonomous Oscillation in Poly(NIPAM-co- $\beta$-CD) Grafted Membrane}

An autonomous complexation/decomplexation phenomenon in poly(NIPAM-co- $\beta$-CD) grafted membrane was observed using UV-vis spectrometer with a conventional quartz cell (light pass $=10$ $\mathrm{mm})$. Poly(NIPAM-co- $\beta$-CD) grafted membrane was cut into pieces of $0.5 \times 0.5 \mathrm{~cm}^{2}$, and immersed into ANS aqueous solution ( $56 \mu \mathrm{M}, 4 \mathrm{ml})$ inside the measurement cell. PTFE mesh was introduced inside the cell to avoid the contact of membrane and UV light as shown in Figure 3. After equilibrating ANS aqueous solution and the membrane at $20^{\circ} \mathrm{C}$ for $8 \mathrm{hr}$, cell temperature was drastically increased and held at $45{ }^{\circ} \mathrm{C}$, and subsequent UV absorbance change of ambient ANS solution was observed. UV measurement of the phenomenon was carried out at wavelength of $265 \mathrm{~nm}$, which is near the maximum absorbance of ANS.

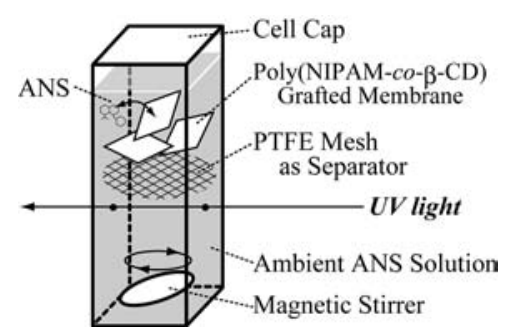

Figure 3. Construction of UV cell for observation of autonomous oscillatory phenomenon of poly (NIPAM-co- $\beta$-CD) grafted membrane.

\section{Results and Discussion}

3.1 Synthesis of Poly(NIPAM-co- $\beta$-CD) Grafted Membrane

As shown in FT-IR spectrum of Figure 4(a), successful preparation of the poly(NIPAM-co-AAc) grafted membrane was confirmed by the peak appearance of $1710 \mathrm{~cm}^{-1}$, from carboxyl group, and $1520-1550 \mathrm{~cm}^{-1}$, from acrylamide group, which are not in polyethylene substrate.

From the same figure, success in the subsequent preparation of the poly(NIPAM-co-NHS) grafted membrane was also confirmed by the increase in peak at $1650 \mathrm{~cm}^{-1}$, from ester group, and 1735, $1770 \mathrm{~cm}^{-1}$, from imide group, while decrease in $1710 \mathrm{~cm}^{-1}$, carboxyl group of AAc. From the result of NHS hydrolytic detachment test, $64 \mathrm{~mol} \%$ of the AAc moiety in grafted polymer was found to be converted into NHS group $(y /(x+y)=64 \%)$.

Figure 4(b) shows the FT-IR of the poly(NIPAM-co- $\beta$-CD) grafted membrane after reacted with isopropylamine. Introduction of $\beta$-CD was confirmed by the FT-IR observation at 1040 $\mathrm{cm}^{-1}$, from hydroxyl group, and $1080 \mathrm{~cm}^{-1}$, from ether group. Decrease in peak height at $1710 \mathrm{~cm}^{-1}$, from carboxyl group, and increase in the peak height at $1550 \mathrm{~cm}^{-1}$, from acrylamide group also confirm the introduction of $\beta-C D$ and additional NIPAM. Ratio of $\beta$-CD was calculated as high as $4.6 \mathrm{~mol} \%$ to the total grafted polymer. Final molar ratio of NIPAM : AAc : $\beta$-CD in the grafted polymer was determined to be $84.7: 10.7: 4.6$ (= $\left.1-x^{\prime}-y^{\prime}: x^{\prime}: y^{\prime}\right)$. Due to the additional introduction of NIPAM via IPAm reaction with NHS moiety, considerably low AAc content was attained compared with the previous studies for the present high CD introduction.

\subsection{Observation of Nonlinear Autonomous Phe- nomenon as Membrane Material}

Using the poly(NIPAM-co- $\beta$-CD) grafted membrane, nonlinear oscillatory complexation/ decomplexation phenomenon of ANS/CD was observed. Poly(NIPAM-co- $\beta$-CD) grafted membrane having 


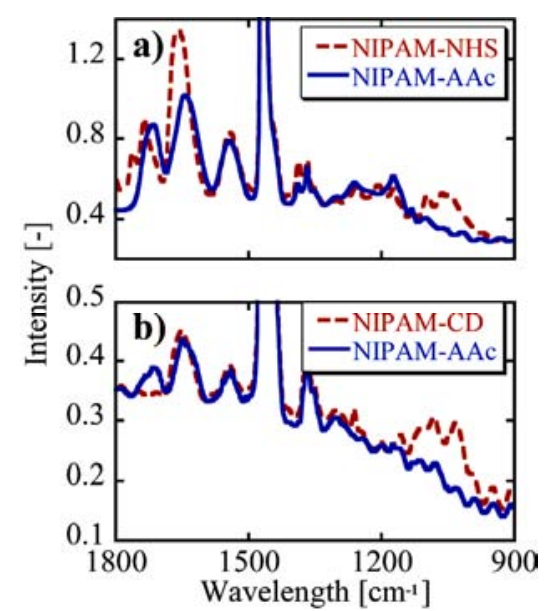

Figure 4. IR spectra of (a) poly(NIPAM-co-AAc) and poly(NIPAM-co-NHS) grafted membrane, and (b) poly(NIPAM-Co-AAc) and poly(NIPAM-Co- $\beta$-CD) grafted membrane.

grafting degree of $0.13 \mathrm{mg} \mathrm{cm}^{-2}$ was used for the following investigation. The time course of UV absorbance of the ambient ANS solution, after the initial temperature shooting from $25^{\circ} \mathrm{C}$ to $45^{\circ} \mathrm{C}$, is shown in Figure 5. More than one cycle of autonomous oscillation was successfully demonstrated in the membrane system. Initial increase in absorbance means ANS concentration increase in the ambient solution, and thus, means ANS detachment from CD moiety inside the membrane. This initial feature is coincided with the previous study for linear polymer of poly(NIPAM-co- $\beta$-CD). Also slower kinetics of ANS detachment process and faster kinetics of ANS recomplexation process again agree with the previous study. Amplitude of the oscillation becomes weak by one cycle, and after two and half cycle, the oscillation ceased.

It is quite interesting although this system is not open system, such an autonomous oscillation is observed. Also it is noteworthy that such oscillation did not occur in the case of the membrane having higher grafting degree. These facts suggest that mass transfer in the membrane pore is crucial in the membrane system, compared with homogeneous linear polymer solution system. However, oscillation continues only two and half cycle due to equilibration of the system after some time. In order to realize successive oscillation, we are currently engaged in a study of open system with ANS concentration gradient through the membrane.

\section{Conclusion}

We have developed a novel method to immobilize poly(NIPAM-co- $\beta$-CD) with high content of $\mathrm{CD}$ and relatively low content of other comonomer

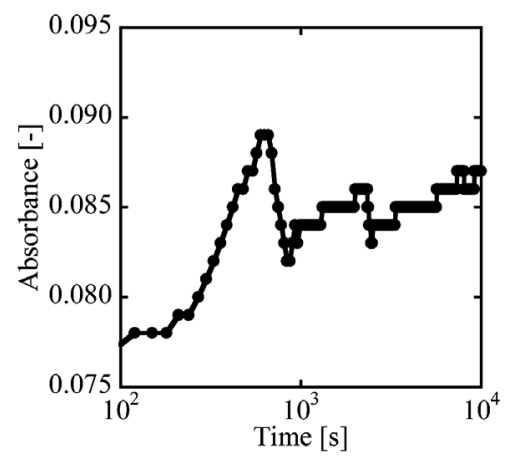

Figure 5. Coordinative oscillatory phenomenon observed by ambient UV absorbance of ANS solution with poly(NIPAM- co- $\beta$-CD) grafted membrane.

onto pore surface of porous membrane. By using the prepared membrane, we have succeeded in demonstrating the repeated autonomous adsorption-desorption phenomenon induced by molecular recognition for the first time. In the future, longterm oscillatory system via open system would be realized, and this could be a model system of living body, and would be applied to new intelligent pulsatile devices actuated by molecular recognition.

\section{References}

1. A. N. Zaikin and A. M. Zhabotinsky, Nature, 225 (1970) 535.

2. N. Minoura, S. Aiba, and Y. Fujiwara, J. Am. Chem. Soc., 115 (1993) 5902.

3. A. Higuchi and M. Hara, J. Phys. Chem., 100 (1996) 2183.

4. G. P. Misra and R. A. Siegel, J. Controlled Release, 81 (2002) 1.

5. A. P. Dhanarajan, G. P. Misra, and R. A. Siegel, J. Phys. Chem. A, 106 (2002) 8835.

6. T. Ito and T. Yamguchi, Angew. Chem. Int. Ed., 45 (2006) 5630.

7. H. Ohashi, Y. Hiraoka, S.-I. Nakao, and T. Yamaguchi, Macromolecules, 39 (2006) 2614.

8. M. Yanagioka, H. Kurita, T. Yamaguchi, and S.-I. Nakao, Ind. Eng. Chem. Res., 42, (2003) 380.

9. M. Yang, L.-Y. Chu, H.-D. Wang, R. Xie, H. Song, and C. H. Niu, Adv. Funct. Mater., 18 (2008) 652.

10. R. Xie, S.-B. Zhang, H.-D. Wang, M. Yang, P.-F. Li, X.-L Zhu, and L.-Y. Chu., J. Membr. Sci., 326 (2009) 618.

11. M. Yang, L.-Y. Chu, R. Xie, and C. Wang, Macrommol. Chem. Phys., 209 (2008) 204.

12. T. Yamaguchi, S.-I. Nakao, and S. Kimura, Macromolecules, 24 (1991) 5522.

13. M. Suzuki, A. Kishida, H. Iwata, and Y. Ikada, Macromolecules, 19 (1986) 1804. 